\title{
Oxygen Content of Twin Domains in YBCO by Geometric Phase Analysis
}

\author{
C.L. Johnson* and Y. Zhu* \\ *Center for Functional Nanomaterials, Brookhaven National Lab, Bldg. 480, Upton, NY 11973
}

The structure of the high-temperature superconductor $\mathrm{YBa}_{2} \mathrm{Cu}_{3} \mathrm{O}_{7-\delta}(\mathrm{YBCO})$ has been extensively studied [1]. Twin boundaries oriented parallel to $<110>$ planes are ubiquitous in YBCO, and accommodate strain induced by the transformation from the oxygen-depleted tetragonal phase to the oxygen-enriched orthorhombic phase. We employed high-resolution transmission electron microscopy (HRTEM) and geometric phase analysis (GPA) to measure orthorhombicity [as characterized by the ratio of $a_{o}$ to $\left.b_{o}(a / b)\right]$ within adjacent domains in twinned YBCO samples. In the absence of doping by other elements, $a / b$ can be directly related to oxygen content ( $\delta$; Fig. 1$)$, which affects the superconducting transition temperature and superconductivity of the material. Commonly, the average $\mathrm{a} / \mathrm{b}$ in a bulk YBCO sample or film has been determined by X-ray diffraction. However, some studies have reported nanoscale inhomogeneities in local oxygen content $[2]$.

GPA is an image processing technique that combines real-space and Fourier-space information to quantitatively map the phase of lattice fringes in HRTEM images [3]. The phase is measured with respect to a reference lattice, which is characterized by an average reciprocal-space vector $\mathbf{g}_{\mathrm{hkl}}$. Using $\mathbf{g}_{100}$ and $\mathbf{g}_{010}$ determined for large selected areas within several individual twin domains, the average $\mathrm{a} / \mathrm{b}$ values for the domains were calculated. All images were corrected for projector-lens distortions prior to the determination of $a / b$ [4]. Figure $2 a$ shows a typical HRTEM image of twinned YBCO in the [001] orientation. The maps of the modulus of $\mathbf{g}_{100}$ and $\mathbf{g}_{010}$ (Figs. $2 \mathrm{~b}$ and $2 \mathrm{c}$ ) show the change in lattice parameter across the boundary. By dividing the average values of $\mathbf{g}_{100}$ by $\mathbf{g}_{010}, \mathrm{a} / \mathrm{b}$ is determined. GPA provides a flexible approach for measuring $\mathrm{a} / \mathrm{b}$ within single domains. Furthermore, because the analysis is performed on HRTEM images, the spatial relationships of the domains are known. Therefore comparisons can be made between adjacent domains.

Initial results from analysis of 33 twin domains occurring in 6 different grains yielded the full range of possible $\mathrm{a} / \mathrm{b}$ values ( 0.983 to 1.00$)$. More importantly, different $\mathrm{a} / \mathrm{b}$ values commonly occur in adjacent domains. Using the relationship between $\mathrm{a} / \mathrm{b}$ and $\delta$ determined from $\mathrm{X}$-ray studies, the $\mathrm{O}$ content of the individual domains can be inferred. Inhomogeneities in O content are likely to play an important role in the performance of YBCO as a practical superconducting material.

References

[1] Z-X. Cai and Y. Zhu, Microstructures and Structural Defects in High-Temperature Superconductors, World Scientific, Singapore, 1998.

[2] P.A. Midgley et al., Philos. Mag. A 66 (1992) 237; E.J. Williams and W.M. Stobbs, Philos. Mag. A 68 (1993) 1; J.C. Barry and J.A. Alarco, J. Micros. 202 (2001) 495.

[3] M.J. Hÿtch et al., Ultramicroscopy 74 (1998) 131.

[4] F. Hüe et al. J. Electron Micros. 54 (2005) 181.

[5] J.D. Jorgensen et al. Phys. Rev. B 36 (1987) 5731.

[6] This work was supported by the Division of Materials Sciences, Office of Basic Energy Sciences, U.S. Department of Energy. 


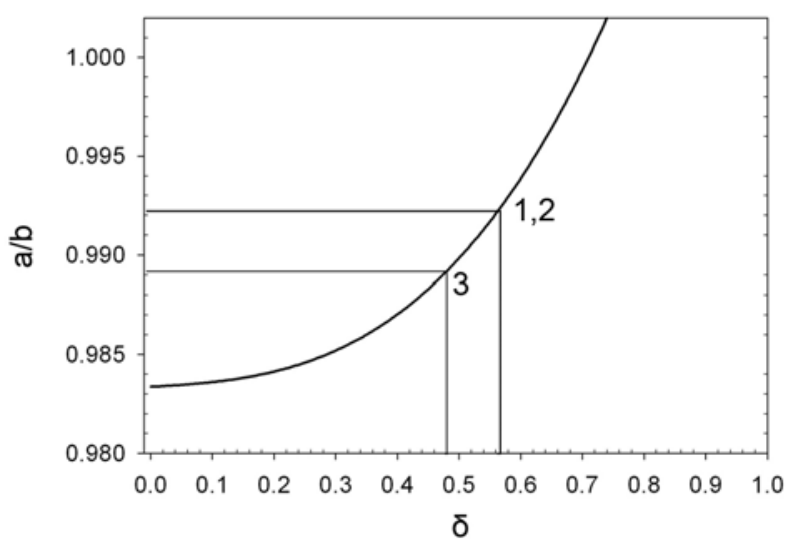

FIG. 1. Plot of $\mathrm{a} / \mathrm{b}$ versus $\delta$ from neutron-scattering measurements [5]. ' 1 ', '2', and '3' indicate where the $\mathrm{a} / \mathrm{b}$ ratios determined from regions 1,2 , and 3 (Fig. 2) fall on the plot.

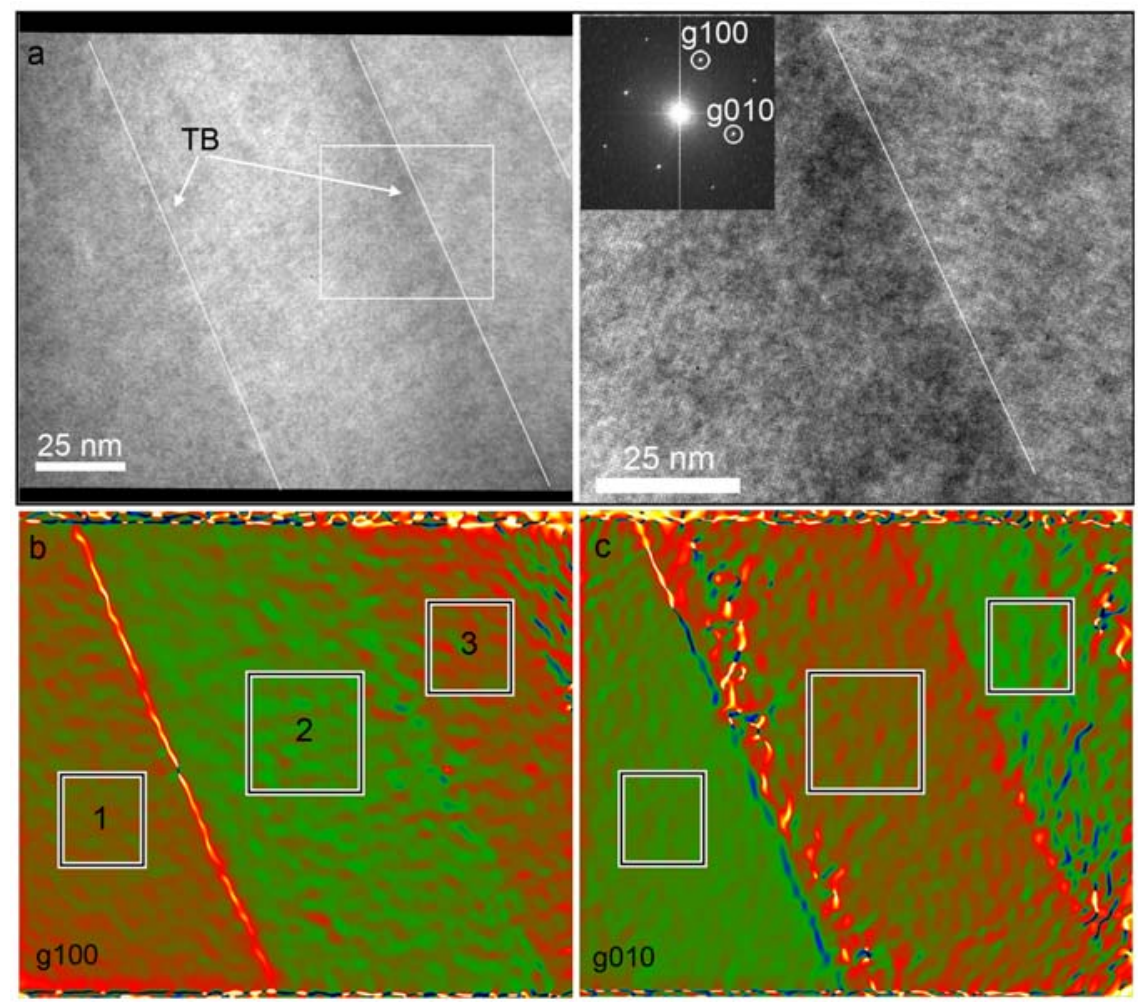

FIG. 2. (a) HRTEM image of YBCO in the [001] zone-axis orientation. Twin boundaries parallel to the (101) planes are indicated by white lines. Enlargement (right) of the boxed region in (a) shows the (100) and (010) lattice planes on either side a twin boundary. Maps of the moduli of $\mathbf{g}_{100}$ (b) and $\mathbf{g}_{010}$ (c) show the change in lattice parameter across the twin boundaries. The boxes show the regions used to measure $a / b$ for each domain. For example $a / b$ for the farthest left domain is determined by dividing the average value within region 1 of the $\mathbf{g}_{100}$ map by the average value in the same region of the $\mathbf{g}_{010}$ map. In this case, the $\mathrm{a} / \mathrm{b}=0.992$ in region 1 compared with 0.989 in region 3 , which correspond to $\mathrm{d}$ values of 0.56 and 0.48 , respectively. 